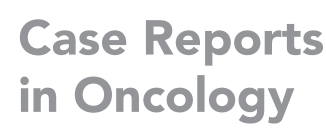

Case Reports

in Oncology

\title{
Changing the Prognosis of Metastatic Cervix Uteri Adenosquamous Carcinoma through a Multimodal Approach: A Case Report
}

\author{
Mihai Teodor Georgescu ${ }^{a}$ Dragos Eugen Georgescu ${ }^{b}$ \\ Teodor Florin Georgescu ${ }^{b}$ Luiza Georgia Serbanescu ${ }^{a}$ \\ ath Department (Radiology, Oncology, Hematology), "Carol Davila" University of Medicine \\ and Pharmacy, Bucharest, Romania; b10th Department (General Surgery), "Carol Davila" \\ University of Medicine and Pharmacy, Bucharest, Romania
}

\author{
Keywords \\ Adenocarcinoma · Cervical cancer · Radiation therapy · Chemotherapy · Surgery
}

\section{Abstract}

Adenocarcinoma and adenosquamous carcinoma (AS) are 2 rare histological types of cervix uteri cancer constituting almost $20 \%$ of all cervix cancers, leading to a lack in patient management guidelines. We report the case of a 32-year-old woman with an oligometastatic cervix AS for which a multimodal treatment approach was used. Despite the patient's bad prognosis, a complete response was achieved, which further resulted in excellent local control and prolonged survival. This case report serves the purpose of encouraging multidisciplinary team work and out-of-the-box thinking that should result in an individualized treatment for rare cancer subtypes.

\section{Introduction}

Cytological screening and human papillomavirus vaccination resulted in a marked decline in cervix carcinoma incidence and mortality rates worldwide in the past decade [1]. Following stage, young age has proved to be one of the most important prognostic factors in cervical cancer [2]. However, this decrease has been shown to be mainly attributable to a decrease in squamous cell carcinoma (SCC), resulting in an increase in the inci- 


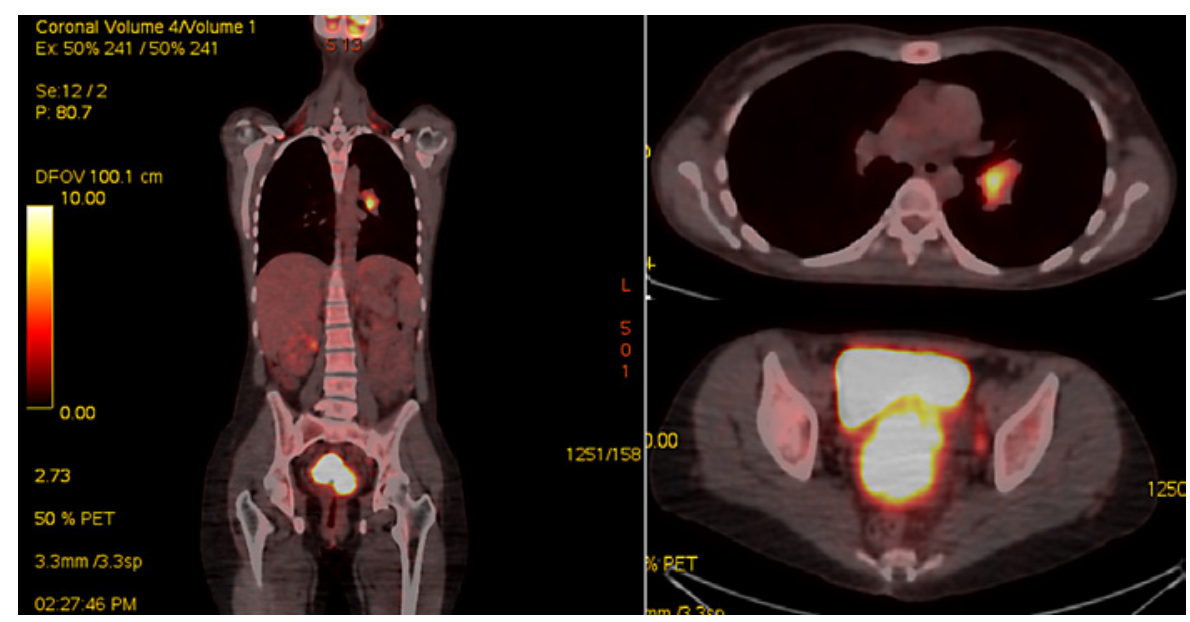

Fig. 1. PET-CT scan result.

dence of less frequent subtypes of cervical cancer, such as adenocarcinoma (AC) and adenosquamous subtypes. With an increase in AC rates among cervical cancer patients, more literature data appeared focusing on this histological subtype [3-5]. This led to the conclusion that cervix uteri AC and SCC are completely different in epidemiology, prognostic factors, patterns of failure and treatment response following treatment; therefore, currently new treatment strategies adapted to AC cervix cancer subtype are explored [3]. Considering all this and the fact that epidemiological data suggest that the median survival time for metastatic cervix cancer patients is only 8-13 months, mainly due to the lack of standard treatment $[6,7]$, we report the case of an adenosquamous carcinoma (AS) of the cervix in a young patient.

\section{Case Report/Case Presentation}

We present the case of a 32-year-old female with no oncological family history, nonsmoker, nonalcoholic, who was referred to our hospital due to vaginal bleeding, pelvic pain, and dispareunia. At the presentation interview, we found out that the patient's menarche had started at 10 years of age, she had 2 children, both born by cesarean section at her request, no abortion history, with dysmenorrhea and metrorrhagia that had developed in the last 8 months. The patient presented at the clinical exam with an European Cooperative Oncology Group (ECOG) performance status of 1 , normal body mass index, afebrile, without skin pallor. The pelvic examination revealed signs of recent bleeding (intravaginal blood clots) and a tumoral mass totally encompassing the cervix uteri and extending in both parametria and in the upper third of the posterior vaginal wall. No enlarged lymph nodes or signs of rectal invasion were identified by clinical exam. The pathology result confirmed the diagnosis of AS (Fig. 1). Positron emission tomography/computed tomography (PET-CT) showed a focus of high 18F-fluorodeoxyglucose uptake in the patient's cervix uteri tumor, which was extending in both parametria, in the pelvic and lomboaortic lymph nodes, and in the left lung hilum. The patient underwent thoracic surgery with bronchoscopy and CT-guided pulmonary hilum tumor biopsy. The immunohistochemistry result confirmed the lymphatic adenosquamous cell metastasis diagnosis, resulting in a IVB stage. Serological tumor markers were evaluated indicating 3-5 times increased values for SCC, CA125, CEA, and CA15.3.

\section{Karger'}





Fig. 2. VMAT treatment plan isodose line for 95\% prescribed dose (PTV45.9-colour washed blue, PTV54colour washed orange).

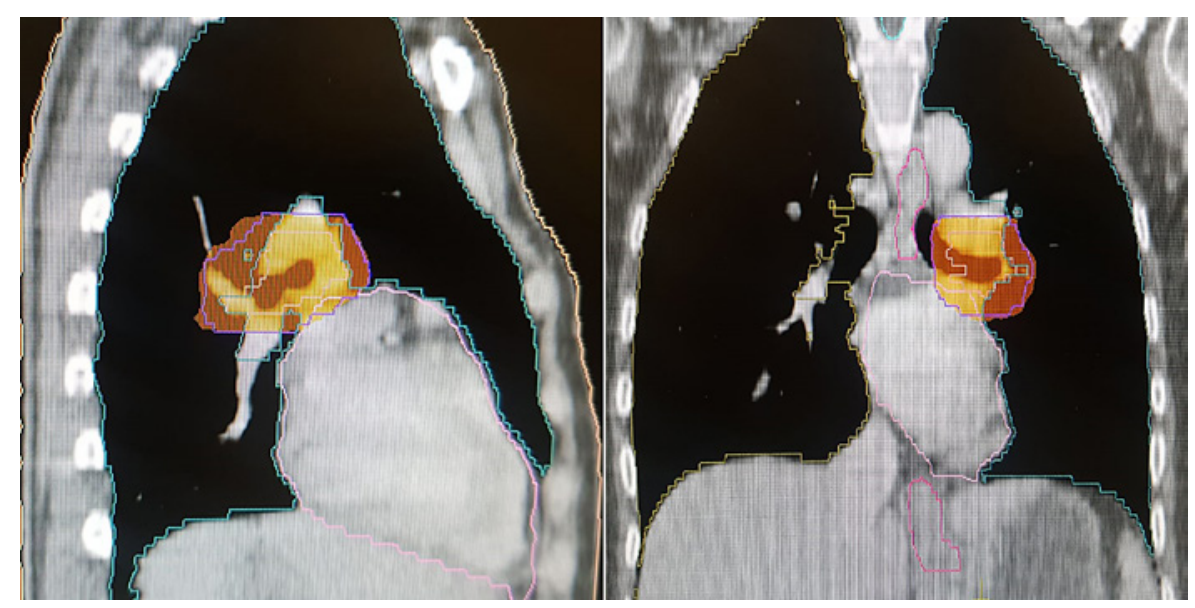

Fig. 3. SBRT treatment plan dose distribution.

Volume-modulated arc-therapy (VMAT) has been administered in 27 fractions up to a total dose of 45.9 Gy delivered to the pelvic and lomboaortic lymph nodes and up to 54 Gy delivered to the primary tumor using a simultaneous integrated boost (SIB) technique. Radiation dose distribution in the target volumes and its relationship with the organs of risk is presented in Figure 2. Following VMAT, intracavitary brachytherapy was delivered in 3 sessions up to a total dose of 21 Gy using a 7-Gy fractionation schedule. Three cycles of concurrent chemotherapy with paclitaxel and carboplatin were administered during radiotherapy and brachytherapy, starting on day 1 of radiotherapy.

At the 2-month follow-up imaging, tumor and lymph nodes shrinkage was observed, except for the stable left hilum lymphatic metastasis. Imaging data were correlated with tumor marker normalization; therefore, the patient was referred for surgical evaluation. Radical hysterectomy with 18 pelvic and lomboaortic lymph node excision was performed. 
After immunohistochemistry, residual disease was identified in the cervix uteri and in 1 of the pelvic lymph nodes.

The patient underwent another 4 cycles of chemotherapy with paclitaxel and carboplatin. During chemotherapy, stereotactic body radiotherapy (SBRT) up to a total dose of 40 Gy using a 10-Gy/fraction schedule was administered to the left lung hilum adenopathy as presented in Figure 3.

At postchemoradiotherapy follow-up, no signs of tumoral mass or abnormal tumoral markers were identified. Imaging and biochemical tests were stable at the latest follow-up, 31 months following the last treatment. Referring to treatment side effects, despite the aggressiveness of the treatment schedule, no genitourinary, gastrointestinal, hematologic, or respiratory toxicity was reported.

\section{Discussion}

The majority of the epidemiological survival data currently available confirm that the histological type is an important prognostic factor for cervix cancer patients [8], AC subtypes carrying a worse prognosis compared to SCC, with more than $10 \%$ difference in 5 -year overall survival rates $[1,9]$. Other significant survival prognostic factors for cervix AC have been identified to be FIGO stage and lymph node status. Initially, Baalbergen et al. [10] demonstrated that for a stage II FIGO status, as our patient has been clinically staged, 5-year overall survival rates are $37 \%$. However, current literature data $[10,11]$ suggest that positive lymph nodes decrease survival rates close to $10 \%$. Based on previous data, we expect lower than $10 \%$ survival rates for patients in the metastatic stage, such as our patient. Also, referring to the pattern of dissemination, a number of studies [10-13] demonstrated higher rates of distant metastasis for AC histology, with an additional increased risk for AS subtypes $[12,13]$. One striking difference between currently reported literature data and our case report is the metastatic site. Current literature data suggest that cervix uteri AS/AC are associated with higher rates of ascites, abdominal carcinomatosis, and para-aortic and supraclavicular spread $[5,9,14]$. However, our patient presented with a biopsy-proven left pulmonary hilum metastasis.

Due to a lack of tumor specificity, usually, serum tumoral markers are not evaluated in cervix uteri cancer. However, after a literature review $[15,16]$, we observed that the initially increased tumor marker (CEA, CA125) values we found are usually correlated with the AC histological type. Usually, SCC tumor marker has been linked to the SCC histological cervix cancer type. We consider that this increase in both AC and SCC subtypesspecific tumor markers in our patient is due to the AS histological type. Also particular to our case is the increase in CA15.3 tumor marker, which is usually associated with breast cancer.

Regarding radiotherapy response of cervix uteri AC/AS and local tumor control, the GOG92 study update [17] suggested that the addition of radiation to the treatment schedule is more effective for patients with this histology compared to SCC types in terms of local control and recurrence rates. Prolonging cervix cancer treatment time beyond 56 days proved to be associated with lower locoregional control and overall survival rates; therefore, the use of SIB treatment delivery is recommended in cases where a radiation boost in scheduled [18]. This was the situation for our patient, available data suggesting that for locally advanced disease total dose escalation is necessary.

Brachytherapy was administered using a tandem and ovoid applicator. Previously published studies $[19,20]$ reported a high influence of uterine position on healthy surrounding tissues regarding radiation exposure and also that initial uterine orientation can change

\section{Karger's}


following external beam radiotherapy. Therefore, and in accordance with current literature recommendations [21], CT-based image-guided brachytherapy was used for our patient in order to obtain a safe and controlled administration of the prescribed radiation dose.

In oligometastatic settings, SBRT represents a pertinent treatment option for the metastatic site, current literature data suggesting that it can replace classic surgical excision in multiple cases, especially due to low toxicity rates and comparable survival outcomes [22]. Also, for our patient, due to the metastatic site in the lung hilum, SBRT was considered the most suitable treatment approach.

The addition of surgery in the treatment of cervix uteri AC/AS is another difference compared to SCC histology, for which in most stages the curative approach consists of chemoradiotherapy. Certain literature data suggest that in some tumor types (e.g., breast cancer, colorectal) removal of the primary in a patient who has stable metastatic disease may improve prognosis and survival [23]. A study form Landoni et al. [24] confirmed the benefits to overall survival and local control by the addition of surgery to radiotherapy in AC/AS cervix uteri patients. However, given the rarity of these tumors, this approach is still debatable.

Despite the lack of data regarding response to different chemotherapy regimens of AC/ AS cervix cancer in the metastatic stage, the results are encouraging regarding overall response rates with certain chemotherapy drugs. Platinum-based chemotherapy regimens remain the mainstay of the systemic treatment independent of the histological type. Existing literature data $[1,25,26]$ suggest that for the non-SCC types taxanes should be added to the chemotherapy regimen. Also, the same data suggest that chemotherapy should be continued in the adjuvant setting in order to decrease distant relapse rates in these patients. For our patient, carboplatin was preferred to cisplatin considering nephrotoxicity side effect, for which there was an increased risk due to lomboaortic lymph nodes irradiation. The chemotherapy regimen increased radiotherapy bone marrow toxicity risk; therefore, pelvic and lumbar bone marrow were contoured as an organ at risk and dose constraints were used according to the current literature [27] during treatment planning.

\section{Conclusion}

As for any metastatic cancer, also for AC and AS of the cervix uteri the aim of the treatment should be to decrease distant failure and local relapse rates. Based on patient performance and hematologic status, current case report results recommend the use of taxane and platinum chemotherapy regimens concurrent with radiotherapy for metastatic AC and AS of the cervix uteri. In case of tumor complete/partial response or stable disease, curative surgery should be performed. Following curative treatment, it is recommended to add adjuvant chemotherapy regimens to the treatment schedule. Regarding oligometastatic disease, considering current progress in radiotherapy delivery, our opinion is that surgical excision should be replaced by SBRT of the metastatic site.

\section{Statement of Ethics}

All procedures followed have been performed in accordance with the ethical standards laid down in the 1964 Declaration of Helsinki and its later amendments. Written informed consent for publishing this case, including images, was obtained from the patient.

\section{Karger's}




\section{Conflict of Interest Statement}

The authors have no conflicts of interest to declare.

\section{Funding Sources}

There are no financial conflicts of interest to disclose.

\section{Author Contributions}

Dr. Mihai T. Georgescu contributed by conceptualization, investigation, resources, writing (original draft preparation), and project administration. Dr. Dragos E. Georgescu contributed by conceptualization, investigation, writing (review and editing), and project supervision. Dr. Teodor F. Georgescu contributed by formal analysis, investigation, literature analysis, and writing (review and editing). Dr. Luiza G. Serbanescu contributed by conceptualization, investigation, resources, writing (original draft preparation), and project administration. All authors contributed equally and in a substantive manner to the conception and content of the article.

\section{References}

1 Gien LT, Beauchemin MC, Thomas G. Adenocarcinoma: a unique cervical cancer. Gynecol Oncol. 2010;116(1): 140-6.

2 Meanwell CA, Kelly KA, Wilson S, Roginski C, Woodman C, Griffiths R, et al. Young age as a prognostic factor in cervical cancer: analysis of population based data from 10,022 cases. Br Med J (Clin Res Ed). 1988;296(6619): 386-91.

3 Fujiwara K, Monk B, Devouassoux-Shisheboran M. Adenocarcinoma of the uterine cervix: Why is it different?. Curr Oncol Rep. 2014;16(12):416.

4 Smith HO, Tiffany MF, Qualls CR, Key CR. The rising incidence of adenocarcinoma relative to squamous cell carcinoma of the uterine cervix in the United States: a 24-year population-based study. Gynecol Oncol. 2000; 78(2): 97-105.

5 Bray F, Carstensen B, Møller H, Zappa M, Zakelj MP, Lawrence G, et al. Incidence trends of adenocarcinoma of the cervix in 13 European countries. Cancer Epidemiol Biomarkers Prev. 2005;14(9):2191-9.

6 Li H, Wu X, Cheng X. Advances in diagnosis and treatment of metastatic cervical cancer. J Gynecol Oncol. 2016; 27(4):e43.

7 van Meir H, Kenter GG, Burggraaf J, Kroep JR, Welters MJ, Melief CJ, et al. The need for improvement of the treatment of advanced and metastatic cervical cancer, the rationale for combined chemo-immunotherapy. Anticancer Agents Med Chem. 2014;14(2):190-203.

8 Eifel PJ, Burke TW, Morris M, Smith TL. Adenocarcinoma as an independent risk factor for disease recurrence in patients with stage IB cervical carcinoma. Gynecol Oncol. 1995;59(1):38-44.

9 Lee KBM, Lee JM, Park CY, Lee KB, Cho HY, Ha SY. What is the difference between squamous cell carcinoma and adenocarcinoma of the cervix? A matched case-control study. Int J Gynecol Cancer. 2006;16(4):1569-73.

10 Baalbergen A, Ewing-Graham PC, Hop WC, Struijk P, Helmerhorst TJ. Prognostic factors in adenocarcinoma of the uterine cervix. Gynecol Oncol. 2004;92(1):262-7.

11 Berek JS, Hacker NF, Fu YS, Sokale JR, Leuchter RC, Lagasse LD. Adenocarcinoma of the uterine cervix: histologic variables associated with lymph node metastasis and survival. Obstet Gynecol. 1985;65(1):46-52.

12 Lea JS, Coleman RL, Garner EO, Duska LR, Miller DS, Schorge JO. Adenosquamous histology predicts poor outcome in low-risk stage IB1 cervical adenocarcinoma. Gynecol Oncol. 2003;91(3):558-62.

13 Gallup DG, Harper RH, Stock RJ. Poor prognosis in patients with adenosquamous cell carcinoma of the cervix. Obstet Gynecol. 1985;65(3):416-22.

14 Drescher CW, Hopkins MP, Roberts JA. Comparison of the pattern of metastatic spread of squamous cell cancer and adenocarcinoma of the uterine cervix. Gynecol Oncol. 1989;33(3):340-3.

15 Borras G, Molina R, Xercavins J, Ballesta A, Iglesias J. Tumor Antigens CA 19.9, CA 125, and CEA in carcinoma of the uterine cervix. Gynecol Oncol. 1995;57(2):205-11. 


\section{Case Reports in Oncology}

\begin{tabular}{l|l}
\hline Case Rep Oncol 2020;13:1545-1551 \\
\hline DOI: 10.1159/000511564 & $\begin{array}{l}\text { ○ 2020 The Author(s). Published by S. Karger AG, Basel } \\
\text { www.karger.com/cro }\end{array}$ \\
\hline
\end{tabular}

Georgescu et al:: Multimodal Treatment for Cervix Uteri Carcinoma

16 Duk JM, Aalders JG, Fleuren GJ, Krans M, De Bruijn HW. Tumor markers CA 125, squamous cell carcinoma antigen, and carcinoembryonic antigen in patients with adenocarcinoma of the uterine cervix. Obstet Gynecol. 1989;73(4):661-8.

17 Rotman M, Sedlis A, Piedmonte MR, Bundy B, Lentz SS, Muderspach LI, et al. A phase III randomized trial of postoperative pelvic irradiation in stage $1 \mathrm{~B}$ cervical carcinoma with poor prognostic features: follow-up of a Gynecologic Oncology Group study. Int J Radiat Oncol Biol Phys. 2006;65(1):169-76.

18 Cheng JY, Huang EY, Hsu SN, Wang CJ. Simultaneous integrated boost (SIB) of the parametrium and cervix in radiotherapy for uterine cervical carcinoma: a dosimetric study using a new alternative approach. Br J Radiol. 2016;89(1068):20160526.

19 Georgescu MT, Anghel R. Variation in uterus position prior to brachytherapy of the cervix: A case report. J Med Life. 2017;10(1):90-3.

20 Georgescu MT, Moldoveanu VG, Ileanu BV, Anghel R. Dosimetric influence of uterus position in cervix cancer high-dose-rate brachytherapy. Rom J Phys. 2016;61(9-10):1557-66.

21 Small W Jr, Strauss JB, Hwang CS, Cohen L, Lurain J. Should uterine tandem applicators ever be placed without ultrasound guidance? No: a brief report and review of the literature. Int J Gynecol Cancer. 2001;21(5):941-4.

22 Wegner RE, Abel S, Hasan S, Schumacher LY, Colonias A. Stereotactic body radiotherapy (SBRT) for oligometastatic lung nodules: a single institution series. Front Oncol. 2019;9:334.

23 Skandarajah A. Principles of cancer surgery. In: Sabesan S, Olver I, editors. Clinical Oncology for Medical Students. Sydney: Cancer Council Australia; 2014. https://wiki.cancer.org.au/oncologyformedicalstudents/ Clinical_Oncology_for_Medical_Students.

24 Landoni F, Maneo A, Colombo A, Placa F, Milani R, Perego P, et al. Randomised study of radical surgery versus radiotherapy for stage Ib-IIa cervical cancer. Lancet. 1997;350(9077):535-40.

25 Nagao S, Fujiwara K, Oda T, Ishikawa H, Koike H, Tanaka H, et al. Combination chemotherapy of docetaxel and carboplatin in advanced or recurrent cervix cancer: a pilot study. Gynecol Oncol. 2005;96(3):805-9.

26 Kastratis E, Bamias A, Efstathiou E, Gika D, Bozas G, Zorzou P, et al. The outcome of advanced or recurrent non-squamous carcinoma of the uterine cervix after platinum-based combination chemotherapy. Gynecol Oncol. 2005;99(2):376-82.

27 Bao Z, Wang D, Chen S, Chen M, Jiang D, Yang C, et al. Optimal dose limitation strategy for bone marrow sparing in intensity-modulated radiotherapy of cervical cancer. Radiat Oncol. 2019;14(1):118.

\section{Karger's}

